\title{
Soft Pomeron in light of the LHC correlated data
}

\author{
M. Broilo, ${ }^{1, *}$ D. A. Fagundes $\odot^{2, \dagger}$ E. G. S. Luna $\oplus^{3,+}$ and M. Peláez ${ }^{4, \S}$ \\ ${ }^{1}$ Instituto de Física e Matemática, Universidade Federal de Pelotas, 96010-900 Pelotas, \\ Rio Grande do Sul, Brazil \\ ${ }^{2}$ Departamento de Ciências Exatas e Educação, Universidade Federal de Santa Catarina- \\ Campus Blumenau, 89065-300 Blumenau, Santa Catarina, Brazil \\ ${ }^{3}$ Instituto de Física, Universidade Federal do Rio Grande do Sul, Caixa Postal 15051, \\ 91501-970 Porto Alegre, Rio Grande do Sul, Brazil \\ ${ }^{4}$ Instituto de Física, Facultad de Ingeniería, Universidad de la República, \\ J.H. y Reissig 565, 11000 Montevideo, Uruguay
}

(Received 15 December 2020; accepted 4 January 2021; published 20 January 2021)

\begin{abstract}
The LHC has released precise measurements of elastic proton-proton scattering that provide a unique constraint on the asymptotic behavior of the scattering amplitude at high energies. Recent reanalyses of part of these data indicate that the central values of some forward quantities would be different than initially observed. We introduce correlation information between the original and the reanalyzed data sets in a way suitable for a global fitting analysis of all data. The careful treatment of correlated errors leads to much less stringent limits on the $\rho$ uncertainty and sets up the stage for describing the forward data using a scattering amplitude dominated by only crossing-even terms. In the light of these correlated data, we determine the parameters of the soft Pomeron from the Regge theory. We use Born-level and eikonalized amplitudes. In the Born-level case, we estimate the contribution of the double Pomeron exchange, while in the latter case we investigate the role of the eikonalization in both the one- and two-channel models. The role of the proton-Pomeron vertex form and of the nearest $t$-channel singularity in the Pomeron trajectory receives particular attention. We discuss the implications of our results and present predictions for the total cross section and the $\rho$ parameter in proton-proton collisions at LHC and cosmic ray energies.
\end{abstract}

DOI: 10.1103/PhysRevD.103.014019

\section{INTRODUCTION}

Diffractive processes account for a substantial fraction of hadron-hadron total cross sections at high energies. These processes, which include elastic scattering or single- or double-diffractive dissociation, are characterized by the presence of one or more large rapidity gaps, which in turn were, for many years, usually associated with the exchange of a colorless state having the quantum numbers of the vacuum: the Pomeron $(\mathbb{P})$. Recently, the TOTEM experiment at the LHC released measurements at $\sqrt{s}=13 \mathrm{TeV}$ of the ratio real to imaginary parts of the forward scattering amplitude, namely, $\rho=0.09 \pm 0.01$ and $\rho=0.10 \pm 0.01$ [1]. These values, as compared with measurements at lower

\footnotetext{
*mateus.broilo90@gmail.com

†daniel.fagundes@ufsc.br

tuna@if.ufrgs.br

§mpelaez@fing.edu.uy
}

Published by the American Physical Society under the terms of the Creative Commons Attribution 4.0 International license. Further distribution of this work must maintain attribution to the author(s) and the published article's title, journal citation, and DOI. Funded by SCOAP. energies and predictions from a wide variety of phenomenological models, suggest that a crossing-odd elastic term may play a central role in soft interactions at high energies. Since then, an intense debate has centered around the asymptotic nature of the $C$-parity of the scattering amplitude. Specifically, the TOTEM results have triggered an extensive discussion on the question of whether or not the combined behavior of $\sigma_{\text {tot }}$ and $\rho$ at high energies is a manifestation of the so-called Odderon [2], the $C=-1$ partner of the $C=+1$ Pomeron [3]. In the QCD language, the Odderon can be associated to a colorless $C$-odd $t$-channel state with an intercept at or near one $[4,5]$.

However, recent reanalyses of the TOTEM results for the Coulomb-nuclear interference $(\mathrm{CNI})$ region have shown that the values of $\rho$ at $\sqrt{s}=8$ and $13 \mathrm{TeV}$ may be larger than those reported by the TOTEM Collaboration [6,7]. In one of these reanalyses [6], using the same nuclear amplitude used by TOTEM but a modified formula for the CNI term, the value $\rho=0.123 \pm 0.010$ is obtained at $\sqrt{s}=13 \mathrm{TeV}$; in the other [7], by means of a modified version of the nuclear amplitude [8], the values $\rho=0.135$ and $\rho=0.137$ are obtained at $\sqrt{s}=8 \mathrm{TeV}$, whereas the values $\rho=0.133$ and $\rho=0.134$ are obtained at 
$\sqrt{s}=13 \mathrm{TeV}$. We remark at this point that in a global fitting analysis we cannot simply choose any of these results over another, nor choose all the results while neglecting the systematic correlation between them. We must also remember that correlation information is already necessary in dealing with part of the original TOTEM data. For example, the measurements of $\rho$ at $\sqrt{s}=13 \mathrm{TeV}$ were extracted from the same set of differential cross section data and have their central values depending on different physics assumptions. In this paper, we show that it is possible to include correlated systematics and combine all these data in a meaningful way. For this, we apply the standard procedure adopted by the Particle Data Group in treating correlated errors [9]. As we will see, after the inclusion of the correlated systematics, each measurement may be treated as independent and, as a result, averaged as usual with other data.

In this work, we are also interested in the effects of the unitarization on the soft Pomeron parameters. Then after fitting analyses using only Born-level scattering amplitudes, we investigate the consequences of including eikonalization in one-channel models. Finally, we repeat our eikonal analyses taking into account only two-channel amplitudes. It is worth remarking on the fact that, in general, only one of these three approaches is usually adopted as a default, that is, some authors focus specifically on Born-level calculations (by arguing that unitarity violation only occurs far above the LHC energies), while others focus solely on one-channel eikonal amplitudes. The use of different procedures made results difficult to compare. Hence, in this work, we adopt all these approaches, making not only the comparison of the results more transparent, but also allowing us to follow the evolution of the Pomeron parameters during the transition from Born-level to eikonalized amplitudes.

In summary, we have shown that the LHC data, after the introduction of correlation information, provide a unique constraint on the Pomeron parameters and allow us to study its behavior more thoroughly since the contribution of the Pomeron component to $\chi^{2}$ is absolutely dominant in the LHC regime. Moreover, the small value of $\alpha_{\mathbb{P}}^{\prime}$ usually obtained from screened Regge models indicates that the soft Pomeron may be treated perturbatively, since in the Gribov Reggeon calculus the mean transverse momentum of the partons is given by $\left\langle p_{T}\right\rangle=1 / \sqrt{\alpha_{\mathbb{P}}^{\prime}}[10,11]$. This perturbative approach raises the possibility for building a fundamental theory for soft processes based upon QCD. Additionally, screening effects can be calculated in terms of a two-channel eikonal model and again the correlated LHC data are instrumental in determining the effects of the eikonalization on the Pomeron parameters in both the one- and two-channel models. Thus, given the central role that the soft Pomeron plays in strong processes, its close scrutiny continues to be a core task in hadron physics.
The outline of this paper is as follows. In Sec. II, we introduce the Regge formalism used to model Born-level amplitudes. Within this approach, we investigate the single as well as the double Pomeron exchange. In Sec. III, we investigate the role of the eikonalization procedure in Regge amplitudes. We study both one- and two-channel eikonal models. In Sec. IV, we discuss the PDG procedure for treating correlated errors and present our results. In the last section, we draw our results and conclusions.

\section{BORN-LEVEL AMPLITUDES}

In the soft regime, i.e., small $t$ domain, diffractive processes are described by Regge theory [12], in which the high-energy behavior of the scattering amplitude is described by singularities of the amplitude in the complex plane of angular momentum $j$. In the simplest scenario, the diffractive processes are driven by an isolated pole at $j=\alpha(t)$, resulting in an elastic amplitude $\mathcal{A}(s, t)$ written in terms of the Regge pole trajectory $\alpha(t)$, namely, $\mathcal{A}(s, t) \propto s^{\alpha(t)}$. If more than one pole contributes, the elastic scattering amplitude is expressed in the $s$-channel as a descending asymptotic series of powers of $s$,

$$
\mathcal{A}(s, t)=\sum_{i} \gamma_{i}(t) \eta_{i}(t) s^{\alpha_{i}(t)},
$$

where $\gamma_{i}(t)$ is the residue function and $\eta_{i}(t)$ is the signature factor. Each term in (1) represents a specific exchange in the $t$-channel. From the optical theorem, the total cross section reads

$$
\sigma_{\mathrm{tot}}(s)=\sum_{i} 4 \pi g_{i} s^{\alpha_{i}(0)-1}
$$

where $g_{i} \equiv \gamma_{i}(0) \operatorname{Im}\left\{\eta_{i}(0)\right\}$. The Pomeron as it emerges from fits to forward observables is called soft Pomeron. The magnitude of its intercept plays a central role in Regge theory, since the Pomeron is the pole with the largest intercept, originally $\alpha_{\mathbb{P}}(0)=1$. However, in order to describe the observed increase of all hadronic total cross sections with $s$, the Pomeron should have an effective intercept such that $\alpha_{\mathbb{P}}(0)=1+\epsilon$ with $\epsilon>0$. This supercritical intercept value is arrived at by taking into account, in addition to Regge poles, multi-Pomeron cuts in the $j$-plane.

It is well known that good descriptions of forward data up to the Tevatron energy have been obtained by using a linear Pomeron trajectory [13-17], namely, $\alpha_{\mathbb{P}}(t)=1+$ $\epsilon+\alpha_{\mathbb{p}}^{\prime} t$. The energy dependence of the total and diffractive cross sections is driven by $\epsilon$ while $\alpha_{\mathbb{P}}^{\prime}$ determines the energy dependence of the forward slopes. We must note, however, that ZEUS and H1 small- $t$ data for exclusive $\rho$ and $\phi$ photoproduction call forth a rather nonlinear Pomeron trajectory [18]. We shall see that the data with correlated systematics allow us to address more effectively the 
question of linearity versus nonlinearity of the Pomeron trajectory.

The forward Born-level Regge amplitude introduced some time ago by Donnachie and Landshoff has two contributions [13], one representing an effective single Pomeron and the other representing the exchange of the highest-spin meson trajectories $\left(a_{2}, f_{2}, \omega\right.$, and $\left.\rho\right)$. However, more recent analysis has indicated that the assumption of degeneracy of the mesons trajectories is not supported by the forward data [14-16]. The best results are obtained with a Born-level amplitude decomposed into three contributions,

$$
\mathcal{A}_{\mathrm{Born}}(s, t)=\mathcal{A}_{\mathbb{P}}(s, t)+\mathcal{A}_{+}(s, t)+\tau \mathcal{A}_{-}(s, t),
$$

where $\tau$ flips sign when going from $p p(\tau=-1)$ to $\bar{p} p$ $(\tau=+1)$. The term $\mathcal{A}_{\mathbb{P}}(s, t)$ represents the exchange of the Pomeron, $\mathcal{A}_{+}(s, t)$ the exchange of the Reggeons with $C=$ $+1\left(a_{2}\right.$ and $\left.f_{2}\right)$, and $\mathcal{A}_{-}(s, t)$ that of the Reggeons with $C=-1(\omega$ and $\rho)$. Specifically, the amplitude for single exchange is

$$
\mathcal{A}_{i}(s, t)=\beta_{i}^{2}(t) \eta_{i}(t)\left(\frac{s}{s_{0}}\right)^{\alpha_{i}(t)},
$$

$i=\mathbb{P},+,-$, where $\beta_{i}(t)$ is the elastic proton-Reggeon vertex, $\eta_{i}(t)$ is the signature factor, and $\alpha_{i}(t)$ is the Regge pole trajectory. Here $s_{0}$ is a mass scale usually chosen to be $s_{0}=1 \mathrm{GeV}^{2}$. By comparing the Eqs. (1) and (4), it can be seen that the residue function factorizes as $\gamma_{i}(t)=\beta_{i}^{2}(t)$. The signature factor, which completely defines the phase of the scattering amplitude, is given by [12]

$$
\eta_{i}(t)=-\frac{1+\xi e^{-i \pi \alpha_{i}(t)}}{\sin \left(\pi \alpha_{i}(t)\right)},
$$

where $\xi=+1$ for the Pomeron and the Reggeons $a_{2}$ and $f_{2}$, and $\xi=-1$ for the Reggeons $\omega$ and $\rho$. Thus, the $p p$ and $\bar{p} p$ scatterings are described in terms of Pomeron, positive and negative signature Regge exchange. However, in order to simplify the numerical calculations involved in the forthcoming eikonal analyses, we adopt in this work $\eta_{i}(t)=$ $-e^{-i \frac{\pi}{2} \alpha_{i}(t)}$ for even-signature trajectories and $\eta_{i}(t)=i e^{-i \frac{\pi}{2} \alpha_{i}(t)}$ for odd-signature ones [15]. The choice of these simplified signatures does not affect our results since the numerical integrals are strongly dominated by the region where $t$ is very small.

The positive-signature secondary Reggeons $\left(a_{2}\right.$ and $\left.f_{2}\right)$ are taken to have an exponential form for the protonReggeon vertex,

$$
\beta_{+}(t)=\beta_{+}(0) \exp \left(r_{+} t / 2\right),
$$

and to lie on an exchange-degenerate linear trajectory of form

$$
\alpha_{+}(t)=1-\eta_{+}+\alpha_{+}^{\prime} t .
$$

Similarly, the negative-signature secondary Reggeons $(\omega$ and $\rho$ ) are described by the parameters $\beta_{-}(0), r_{-}, \eta_{-}$, and $\alpha_{-}^{\prime}$.

For Pomeron exchange, we investigate two different types of proton-Pomeron vertex and two different types of trajectory, one of which being nonlinear. Our philosophy is, using the standard statistical $\chi^{2}$ test, to evaluate the relative plausibility of these vertices and trajectories in the light of the LHC data, i.e., to consider different combinations of $\beta_{\mathbb{P}}(t)$ and $\alpha_{\mathbb{P}}(t)$, and the effectiveness of these combinations at describing the high-energy forward data. In the first combination, referred to as "BI model," we adopt an exponential form for the proton-Pomeron vertex,

$$
\beta_{\mathbb{P}}(t)=\beta_{\mathbb{P}}(0) \exp \left(r_{\mathbb{P}} t / 2\right),
$$

and a linear Pomeron trajectory,

$$
\alpha_{\mathbb{P}}(t)=\alpha_{\mathbb{P}}(0)+\alpha_{\mathbb{P}}^{\prime} t,
$$

where henceforth we define $\alpha_{\mathbb{P}}(0) \equiv 1+\epsilon$. In the second model, called "BII," we adopt the exponential vertex (8) and the nonlinear Pomeron trajectory [19-22]

$$
\alpha_{\mathbb{P}}(t)=\alpha_{\mathbb{P}}(0)+\alpha_{\mathbb{P}}^{\prime} t+\frac{m_{\pi}^{2}}{32 \pi^{3}} h(\tau),
$$

where

$$
\begin{aligned}
h(\tau)= & -\frac{4}{\tau} F_{\pi}^{2}(t)\left[2 \tau-(1+\tau)^{3 / 2} \ln \left(\frac{\sqrt{1+\tau}+1}{\sqrt{1+\tau}-1}\right)\right. \\
& \left.+\ln \left(\frac{m^{2}}{m_{\pi}^{2}}\right)\right],
\end{aligned}
$$

with $\tau=4 m_{\pi}^{2} /|t|, m_{\pi}=139.6 \mathrm{MeV}$ and $m=1 \mathrm{GeV}$. The nonlinear term in the Pomeron trajectory comes from the nearest $t$-channel singularity (a two-pion loop) [19]. In the above expression, $F_{\pi}(t)$ is the form factor of the pion-Pomeron vertex, for which we take the standard pole expression $F_{\pi}(t)=\beta_{\pi} /\left(1-t / a_{1}\right)$. The coefficient $\beta_{\pi}$ specifies the value of the pion-Pomeron coupling and for this we adopt the additive quark model relation $\beta_{\pi} / \beta_{I P}(0)=2 / 3$. In the third combination, called "BIII model," we adopt the nonlinear Pomeron trajectory (10) and the powerlike form for the proton-Pomeron vertex [20-23]

$$
\beta_{\mathbb{P}}(t)=\frac{\beta_{\mathbb{P}}(0)}{\left(1-t / a_{1}\right)\left(1-t / a_{2}\right)},
$$

where the free parameter $a_{1}$ is the same as the one in the form factor of the pion-Pomeron vertex $F_{\pi}(t)$. The total cross section, the elastic differential cross section, and the $\rho$ parameter are expressed in terms of the amplitude (3), 


$$
\begin{gathered}
\sigma_{\text {tot }}(s)=\frac{4 \pi}{s} \operatorname{Im} \mathcal{A}(s, t=0), \\
\frac{d \sigma}{d t}(s, t)=\frac{\pi}{s^{2}}|\mathcal{A}(s, t)|^{2}, \\
\rho(s)=\frac{\operatorname{Re} \mathcal{A}(s, t=0)}{\operatorname{Im} \mathcal{A}(s, t=0)},
\end{gathered}
$$

where $\mathcal{A}(s, t)=\mathcal{A}_{\text {Born }}(s, t)$.

It is important to notice that the Pomeron intercept $\alpha_{\mathbb{P}}(0)=1+\epsilon$ is an effective power, valid over a limited range of energies; otherwise, the forward amplitude $A(s, t=0)$ would grow so large that unitarity bound would be violated. Thus, the parameter $\epsilon$ represents not only the exchange of a single Pomeron but also $n$-Pomeron exchange processes, $n \geq 2$ [12,24]. These multiple exchanges must tame the rise of $\sigma_{\text {tot }}(s)$ so that the breakdown of unitarity is avoided and, as a consequence, the value of $\epsilon$ should decrease slowly with increasing $s$. The search for a hint of unitarization breaking up to LHC energies can be verified by investigating the role of multiple Pomeron exchanges on the scattering amplitude. Unfortunately, despite the advances in theoretical understanding of the Pomeron in the last four decades, we still do not know how to do it. Nevertheless, there is a consensus that the contribution of the double Pomeron exchange $(\mathbb{P P})$ is negative and has energy dependence $s^{\alpha_{\mathbb{P P}}(t)}$ divided by some function of $\ln s$ [25], where

$$
\alpha_{\mathbb{P P}}(t)=1+2 \epsilon+\frac{1}{2} \alpha_{\mathbb{P}}^{\prime} t .
$$

Thus, the $\mathbb{P P}$ contribution is flatter in $t$ than the single $\mathbb{P}$ exchange, becoming more important for higher values of $t$. In order to estimate an upper bound on the ratio $R \equiv \beta_{\mathbb{P} P}^{2}(0) / \beta_{\mathbb{P}}^{2}(0)$, we add the phenomenological term

$$
\mathcal{A}_{\mathbb{P P}}(s, t)=-\beta_{\mathbb{P} P}^{2}(t) \eta_{\mathbb{P} P}(t)\left(\frac{s}{s_{0}}\right)^{\alpha_{\mathbb{P}}(t)}\left[\ln \left(\frac{-i s}{s_{0}}\right)\right]^{-1}
$$

to the amplitude (3), where $\eta_{\mathbb{P P}}(t)=-e^{-i \frac{\pi}{2} \alpha_{\mathbb{P P}}(t)}$ and $\beta_{\mathbb{P P}}(t)=\beta_{\mathbb{P P}}(0) \exp \left(r_{\mathbb{P}} t / 4\right)$. We include this doublePomeron exchange term in the model BI. This combination is henceforth called $\mathrm{BI}+\mathbb{P P}$ model.

\section{EIKONALIZED AMPLITUDES}

\section{A. One-channel amplitudes}

As it was mentioned in the previous section, in the case of Born-level amplitudes, the breakdown of unitarity can be avoided by introducing the exchange series $\mathbb{P}+$ $\mathbb{P P}+\mathbb{P P P}+\cdots$. Although some general analytic properties of these multiple-exchange terms are known, it is less clear how to carry out a full computation of them. On the other hand, it is well established that eikonalization is an effective procedure to take into account some properties of high-energy $s$-channel unitarity. In practice, the unitarity of the matrix $\mathrm{S}$ in impact parameter $(b)$ representation implies the relation [12]

$$
\operatorname{Im} h(s, b)=|h(s, b)|^{2}+G_{\text {inel }}(s, b),
$$

where $h(s, b)$ is the elastic profile function and $G_{\text {inel }}(s, b)$, known as the inelastic overlap function or shadow profile function, is the contribution from all inelastic channels. The profile function $h(s, b)$ is related to the elastic scattering amplitude $\mathcal{A}(s, t)$ by

$$
\mathcal{A}(s, t)=\int_{0}^{\infty} b d b J_{0}(b \sqrt{-t}) h(s, b) .
$$

In this picture, we can think of the sum over all inelastic channels as forming a shadow, which "generates" elastic scattering. The unitarity relation (18) imposes a limit on the elastic profile function, namely, $0 \leq$ $|h(s, b)|^{2} \leq \operatorname{Im} h(s, b) \leq 1$, while eikonalization enforces the so-called black-disc limit: $\operatorname{Im} h(s, b) \leq 1 / 2$. The upper value $1 / 2$ is due to the requirement of a maximal absorption within the eikonal unitarization, in which $h(s, b)$ is written as

$$
h(s, b)=\frac{i}{2}\left[1-e^{i \chi(s, b)}\right],
$$

with the eikonal function $\chi(s, b)=i \Omega(s, b) / 2$ being a purely imaginary function in the limit $s \rightarrow \infty$. In other words, at high energies, the inelastic contribution, $G_{\text {inel }}$, dominates and the scattering amplitude $\mathcal{A}(s, t)$ is predominantly imaginary. In this regime, $\Omega(s, b) \gg 1$ and $\operatorname{Im} h(s, b)=1 / 2$. The eikonalization scheme prevents the Froissart-Martin bound for $\sigma_{\text {tot }}(s)$ from being violated. The bound follows from the theorem which states that $\sigma_{\text {tot }}(s) \leq C \ln ^{2} s$, as $s \rightarrow \infty$, where $C$ is a constant [26]. The Froissart-Martin bound imposes a strict restriction on the rate of growth of any total cross section. It is worth mentioning that while the Froissart-Martin bound holds for all eikonalized amplitudes studied in this paper, it is not necessarily synonymous with total unitarization: it was shown some time ago that any model for input Pomeron with intercept $\alpha_{\mathbb{P}}(0)>1$ but with linear trajectory is affected by small asymptotic violations of unitarity [27]. We also notice that eikonal unitarization corresponds to one of the two solutions of the unitarity equation

$$
h(s, b)=\frac{1}{2}\left[1 \pm \sqrt{1-4 G_{\text {inel }}(s, b)}\right]
$$

the one with minus sign. Choosing the plus sign in (21), we get the alternative solution [28] 


$$
h(s, b)=\frac{\operatorname{Im} \tilde{\chi}(s, b)}{1-i \tilde{\chi}(s, b)},
$$

where $\tilde{\chi}(s, b)$ is the analogue of the eikonal $\chi(s, b)$. In this approach, $h(s, b)$ may exceed the black disc limit. Thus, we see that different unitarization procedures are possible in $b$ representation. In this paper, we follow with the eikonalization procedure: the eikonal function is related to the Born amplitude (3) by the Fourier-Bessel transform

$$
\chi(s, b)=\frac{1}{s} \int_{0}^{\infty} q d q J_{0}(b q) \mathcal{A}_{\mathrm{Born}}(s, t),
$$

where $t=-q^{2}$; its inverse transform leads to the eikonalized amplitude in $(s, t)$-space,

$$
\mathcal{A}_{e i k}(s, t)=i s \int_{0}^{\infty} b d b J_{0}(b q)\left[1-e^{i \chi(s, b)}\right],
$$

to be used in the calculation of the observables. Hence, the total cross section, the elastic differential cross section, and the $\rho$ parameter are calculated using Eqs. (13)-(15) with $\mathcal{A}(s, t)=\mathcal{A}_{\text {eik }}(s, t)$. In the calculation of the eikonal function (23), the input amplitudes $\left(\mathcal{A}_{\mathrm{Born}}(s, t)\right)$ are simply the ones related to BI, BII, and BIII models. These onechannel eikonal models are referred to, respectively, as OI, OII, and OIII models.

\section{B. Two-channel amplitudes}

As it was mentioned in Sec. I, an effective Pomeron intercept $\alpha_{\mathbb{P}}(0)>1$ is obtained taking into account multiPomeron cuts (moving branch points) in the $j$-plane. These singularities are required in order to assure $s$-channel unitarity. In the models considered in the preceding text, we have not accounted for the possibility of diffractive proton excitation in intermediate states, such as $p \rightarrow N^{*}$. However, it is possible incorporate the $s$-channel unitarity with elastic and a low mass intermediate state $N^{*}$ by using a two-channel eikonal approach. The Good-Walker formalism [20,21,29-31] provides an elegant and convenient form to incorporate $p \rightarrow N^{*}$ diffractive dissociation. In this approach, we introduce diffractive eigenstates $\left|\phi_{i}\right\rangle$ that diagonalize the interaction matrix $\mathrm{T}$ (where $\mathrm{S}=1+i \mathrm{~T}$ ). As a result, the incoming hadron wave functions $|h\rangle$ (in our case the "beam" and "target" proton wave functions) can be written as superpositions of these diffractive eigenstates, namely,

$$
|h\rangle_{\text {beam }}=\sum a_{i}\left|\phi_{i}\right\rangle, \quad|h\rangle_{\text {target }}=\sum a_{k}\left|\phi_{k}\right\rangle .
$$

Since we need at least two diffractive eigenstates, in a twochannel eikonal model, we have $i, k=1,2$. The extension to $n$-channel eikonal models is straightforward; however, it is well known that a two-channel model is sufficient to capture the single- or double-diffractive dissociation behavior very accurately [20-23,32-34]. In this paper, we adopt a two-channel eikonal model in which the Pomeron couplings to the two diffractive eigenstates $k$ are

$$
\beta_{\mathbb{P}, k}(t)=(1 \pm \gamma) \beta_{\mathbb{P}}(t),
$$

i.e., the eigenvalues of the two-channel vertex are $1 \pm \gamma$, where $\gamma \simeq 0.55[21,22]$. This value is in accordance with $p \rightarrow N^{*}$ dissociation observed at CERN-ISR energies, more specifically, it is the value required in order to obtain the experimental value of the cross section for low-mass diffraction measured at $\sqrt{s}=31 \mathrm{GeV}$, namely, $\sigma_{\mathrm{SD}}^{\text {low } M} \simeq 2 \mathrm{mb}$.

Since each amplitude has two vertices, the forward observables are controlled by an elastic scattering amplitude with three different exponents,

$$
\begin{aligned}
\mathcal{A}_{e i k}(s, t)= & i s \int_{0}^{\infty} b d b J_{0}(b q)\left[1-\frac{1}{4} e^{i(1+\gamma)^{2} \chi(s, b)}\right. \\
& \left.-\frac{1}{2} e^{i\left(1+\gamma^{2}\right) \chi(s, b)}-\frac{1}{4} e^{i(1-\gamma)^{2} \chi(s, b)}\right] .
\end{aligned}
$$

In the computation of the eikonal functions employed for calculating the amplitude above, again the input Born-level amplitudes are the ones related to BI, BII, and BIII models. These two-channel eikonal models are referred to, respectively, as TI, TII, and TIII models.

\section{CORRELATED EXPERIMENTAL SYSTEMATICS}

In our analyses, we carry out global fits to forward $p p$ and $\bar{p} p$ scattering data above $\sqrt{s}=10 \mathrm{GeV}$ and to elastic $p p$ differential scattering cross section data at LHC energies. Specifically, we fit to the total cross section $\sigma_{\mathrm{tot}}^{p p, \bar{p} p}$, the ratio of the real to imaginary part of the forward scattering amplitude $\rho^{p p, \bar{p} p}$ and to the elastic differential cross section $d \sigma^{p p} / d t$ at $\sqrt{s}=7,8$, and $13 \mathrm{TeV}$ with $|t| \leq$ $0.1 \mathrm{GeV}^{2}$ (this range for $|t|$ is enough for an appropriate evaluation of $\alpha_{\mathbb{P}}^{\prime}$ ). We use data sets compiled and analyzed by the Particle Data Group (PDG) [9] as well as the recent data at LHC from the TOTEM Collaboration [1,35-42], with the statistic and systematic errors added in quadrature.

The PDG database is currently the standard data source used by most research papers in the field. In order to ensure the consistency between TOTEM and PDG information, we must adopt common criteria for the selection and treatment of data. Following the PDG discussion on treatment of errors, we see that correlated errors are indeed treated explicitly in the presence of results of the form $A_{i} \pm$ $\sigma_{i} \pm \Delta$ that have same systematic errors $\Delta$. As usual, it is possible to average the $A_{i} \pm \sigma_{i}$ and then combine in quadrature the resulting statistical error with the respective $\Delta$. However, the same result can be obtained by averaging 
$A_{i} \pm\left(\sigma_{i}^{2}+\Delta_{i}^{2}\right)^{1 / 2}$, where $\Delta_{i}$ are modified systematic errors given by

$$
\Delta_{i}=\sigma_{i} \Delta\left(\sum \frac{1}{\sigma_{j}^{2}}\right)^{1 / 2}
$$

As pointed out by PDG, this alternative procedure has the advantage that each measurement may be treated as independent and, as a consequence may be averaged in the usual way with other data. We will therefore adopt this procedure in preparing the data set to be fitted.

Let us start with the original TOTEM data, which include the first and second measurements of the total protonproton $(p p)$ cross section at $\sqrt{s}=7 \mathrm{TeV}, \sigma_{\mathrm{tot}}^{p p}=98.3 \pm$ $2.8 \mathrm{mb}$ [35], and $\sigma_{\mathrm{tot}}^{p p}=98.6 \pm 2.2 \mathrm{mb}$ [36] (both using the optical theorem together with the luminosity provided by the CMS [43]); the luminosity-independent measurement at $\sqrt{s}=7 \mathrm{TeV}, \sigma_{\mathrm{tot}}^{p p}=98.0 \pm 2.5 \mathrm{mb}$ [37]; the $\rho$-independent measurements at $\sqrt{s}=7 \mathrm{TeV}$ of $\sigma_{\mathrm{tot}}^{p p}$ and $\rho$ parameter, $\sigma_{\mathrm{tot}}^{p p}=99.1 \pm 4.3 \mathrm{mb}$ and $\rho^{p p}=0.145 \pm 0.091$ [37]; the luminosity-independent measurement at $\sqrt{s}=$ $8 \mathrm{TeV}, \sigma_{\mathrm{tot}}^{p p}=101.7 \pm 2.9 \mathrm{mb}$ [38]; the measurements in the Coulomb-nuclear interference region at $\sqrt{s}=8 \mathrm{TeV}$ of $\sigma_{\text {tot }}^{p p}$ and $\rho$ parameter, $\sigma_{\text {tot }}^{p p}=102.9 \pm 2.3 \mathrm{mb}$ and $\sigma_{\text {tot }}^{p p}=$ $103.0 \pm 2.3 \mathrm{mb}$ (for central and peripheral phase formulations, respectively), and $\rho^{p p}=0.12 \pm 0.03$ [39]; the total cross sections at $\sqrt{s}=8 \mathrm{TeV}, \sigma_{\mathrm{tot}}^{p p}=101.5 \pm 2.1 \mathrm{mb}$, and $\sigma_{\text {tot }}^{p p}=101.9 \pm 2.1 \mathrm{mb}$, obtained from extrapolations of the differential cross section to $t=0$ (for quadratic and cubic polynomials in the exponent, respectively) [41]; the luminosity-independent measurements at $\sqrt{s}=13 \mathrm{TeV}$, $\sigma_{\mathrm{tot}}^{p p}=110.6 \pm 3.4 \mathrm{mb}$, and $\sigma_{\mathrm{tot}}^{p p}=109.5 \pm 3.4 \mathrm{mb}$ [44]; the first extraction of the $\rho$ parameter at $\sqrt{s}=13 \mathrm{TeV}$ (exploiting the Coulomb-nuclear interference), $\rho^{p p}=$ $0.09 \pm 0.01$ and $\rho^{p p}=0.10 \pm 0.01$, and the associated total cross section $\sigma_{\mathrm{tot}}^{p p}=110.3 \pm 3.5 \mathrm{mb}$, obtained from the Coulomb normalization technique [1]; the elastic differential cross section in the intervals $0.377 \leq|t| \leq 2.443 \mathrm{GeV}^{2}$ [40] and $0.00515 \leq|t| \leq 0.235 \mathrm{GeV}^{2}[37]$ at $\sqrt{s}=7 \mathrm{TeV}$, in the interval $6 \times 10^{-4} \leq|t| \leq 0.2 \mathrm{GeV}^{2}$ at $\sqrt{s}=8 \mathrm{TeV}$ [39], and in the interval $0.0384 \leq|t| \leq 3.829 \mathrm{GeV}^{2}$ at $\sqrt{s}=$ $13 \mathrm{TeV}$ [42].

From the $\sigma_{\text {tot }}^{p p}$ data at $\sqrt{s}=7 \mathrm{TeV}$, there is already a hint of some kind of correlation among the measurements. The four measurements taken by the TOTEM group were obtained using the same beam optics configuration, namely, $\beta^{*}=90 \mathrm{~m}$. The optics with this betatron value is very insensitive to variations of the machine parameters and led to very low systematic uncertainties on horizontal and vertical scattering angles [35]. Nevertheless, the value $\sigma_{\text {tot }}^{p p}=98.3 \pm 2.8 \mathrm{mb}$ was obtained from the lower-luminosity run in June 2011, whereas the remaining three values, namely, $\sigma_{\mathrm{tot}}^{p p}=98.6 \pm 2.2 \mathrm{mb}$, $\sigma_{\mathrm{tot}}^{p p}=98.0 \pm 2.5 \mathrm{mb}$, and $\sigma_{\mathrm{tot}}^{p p}=99.1 \pm 4.3 \mathrm{mb}$, were obtained in a dedicated run in October 2011. The October run has resulted in an improved measurement of the $t$-distribution with higher statistics. Thus, although there is no correlation between the June and October measurements, the correlation among the last three values of $\sigma_{\mathrm{tot}}^{p p}$ became clear: they were obtained from the same data, recorded in the same run. Precisely, this type of correlation also occurs in some $\sigma_{\text {tot }}^{p p}$ and $\rho^{p p}$ TOTEM data at $\sqrt{s}=8$ and $13 \mathrm{TeV}$.

The correlation issue also appears from reanalyses of part of the TOTEM data. In one of these works [7], it is observed that a zero in the real part of the nuclear amplitude lies in the CNI region, leading to a positive amplitude at $-t=0$. It has been proved some time ago [45] that in the limit $s \rightarrow \infty$, if the total cross section tends to infinity and the differential elastic cross section tends to zero as $-t \gg 1$, the real part of the even amplitude must change sign near $-t=0$. Since these assumptions correspond to the experimentally observed behavior of the cross sections at high energies, the $\rho$ value obtained by TOTEM assuming a constant real part of the nuclear amplitude near $-t=0$ might be underestimated. Thus, in [7], by analyzing the complete TOTEM elastic differential cross section in the CNI region at $\sqrt{s}=8$ and $13 \mathrm{TeV}$, it is shown that two modified Barger and Phillips (BP) amplitudes [46] appear to describe quite adequately the CNI data. The $\rho$ values obtained from these amplitudes are $\rho^{p p}=0.135$ and $\rho^{p p}=$ 0.137 at $\sqrt{s}=8 \mathrm{TeV}$ as well as $\rho^{p p}=0.133$ and $\rho^{p p}=$ 0.134 at $\sqrt{s}=13 \mathrm{TeV}$. Of course, these results are correlated to the TOTEM ones since the same data set is used in their determinations. The modified BP amplitudes

TABLE I. LHC data used in our global fitting analyses. In the case of correlated data, uncertainties are multiplied by a factor $f_{n} \equiv \sqrt{n}$, where $n$ is the number of correlated quantities.

\begin{tabular}{ccc}
\hline \hline$\sqrt{s}(\mathrm{TeV})$ & $\sigma_{\text {tot }}(\mathrm{mb})$ & $\rho$ \\
\hline 13 & $110.6 \pm\left(3.4 \times f_{5}\right)[44]$ & $0.100 \pm\left(0.010 \times f_{4}\right)[1]$ \\
$109.5 \pm\left(3.4 \times f_{5}\right)[44]$ & $0.133 \pm\left(0.010 \times f_{4}\right)[7]$ \\
$111.8 \pm\left(3.4 \times f_{5}\right)[7]$ & $0.134 \pm\left(0.010 \times f_{4}\right)[7]$ \\
$112.9 \pm\left(3.4 \times f_{5}\right)[7]$ & $0.123 \pm\left(0.010 \times f_{4}\right)[6]$ \\
& $111.4 \pm\left(1.8 \times f_{5}\right)[6]$ & $0.090 \pm\left(0.010 \times f_{3}\right)[1]$ \\
& $110.3 \pm 3.5[1]$ & $0.133 \pm\left(0.010 \times f_{3}\right)[7]$ \\
& & $0.134 \pm\left(0.010 \times f_{3}\right)[7]$ \\
& $102.9 \pm\left(2.3 \times f_{4}\right)[39]$ & $0.120 \pm\left(0.030 \times f_{3}\right)[39]$ \\
& $103.0 \pm\left(2.3 \times f_{4}\right)[39]$ & $0.137 \pm\left(0.030 \times f_{3}\right)[7]$ \\
& $101.6 \pm\left(2.3 \times f_{4}\right)[7]$ & $0.135 \pm\left(0.030 \times f_{3}\right)[7]$ \\
$102.7 \pm\left(2.3 \times f_{4}\right)[7]$ & \\
$101.5 \pm\left(2.1 \times f_{2}\right)[41]$ & \\
$101.9 \pm\left(2.1 \times f_{2}\right)[41]$ & \\
7 & $101.7 \pm 2.9[38]$ & \\
7 & $99.1 \pm\left(4.3 \times f_{3}\right)[37]$ & $0.145 \pm 0.091[37]$ \\
& $98.0 \pm\left(2.5 \times f_{3}\right)[37]$ & \\
& $98.6 \pm\left(2.2 \times f_{3}\right)[36]$ & \\
\hline \hline
\end{tabular}




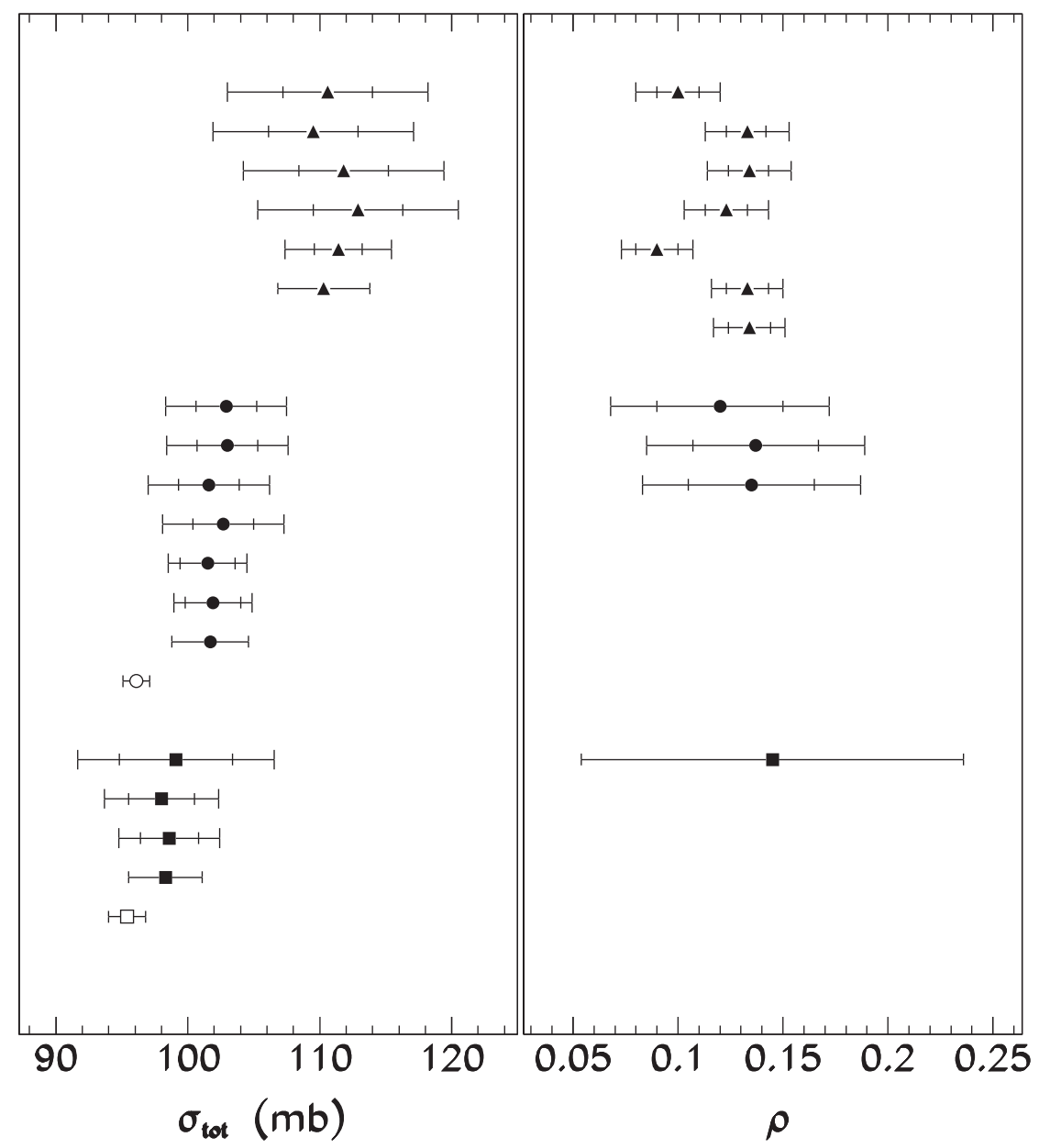

FIG. 1. Set of correlated data used in our global fitting analyses. The inner error bars are the original (published) uncertainties and the outer error bars are the uncertainties after introducing correlation information. The squares, circles, and triangles correspond, respectively, to the energies of 7, 8, and $13 \mathrm{TeV}$. The solid symbols represent the TOTEM data, while the open ones represent the ATLAS results.

also lead to different $\sigma_{\text {tot }}$ values than those obtained by TOTEM: $\sigma_{\text {tot }}^{p p}=102.7 \mathrm{mb}$ and $\sigma_{\text {tot }}^{p p}=101.6 \mathrm{mb}$ at $\sqrt{s}=8 \mathrm{TeV}$, and $\sigma_{\mathrm{tot}}^{p p}=112.9 \mathrm{mb}$ and $\sigma_{\mathrm{tot}}^{p p}=111.8 \mathrm{mb}$ at $\sqrt{s}=13 \mathrm{TeV}$. Once again, we have correlation among these and the TOTEM cross sections result at $\sqrt{s}=8$ and $13 \mathrm{TeV}$. In other reanalysis of the TOTEM data [6], using a modified formula for the CNI term, the values $\rho=$ $0.123 \pm 0.010$ and $\sigma_{\text {tot }}^{p p}=111.4 \pm 1.8 \mathrm{mb}$ are obtained at $\sqrt{s}=13 \mathrm{TeV}$.

Since all results for $\rho^{p p}$ and $\sigma_{\text {tot }}^{p p}$ in [7] are obtained from the same data source used by TOTEM, we would expect their associated errors to be the same as those of the TOTEM papers. Thus, in order to implement a practical procedure for introducing correlation information in our fits, we will consider that the total and systematic errors associated with the results in [7] are the same as those presented by TOTEM. This assumption makes the PDG procedure be consistent, in good approximation, to the rule adopted by experimentalists in accounting for correlation systematics, namely, to the product $\sqrt{n} \delta_{k}$, $k=1, \ldots, n$, where $n$ is the number of correlated results and $\delta_{k}=\sqrt{\sigma_{k}^{2}+\Delta^{2}}$. Thus, for practical reasons, we have adopted this last procedure for the selection and treatment of correlated data. The set of correlated data used in our global fitting analyses is summarized in Table I. In Fig. 1, we show these data with their corresponding uncertainties. The inner error bars are the original (published) uncertainties and the outer error bars are the uncertainties after introducing correlation information.

\section{RESULTS AND CONCLUSIONS}

In all the fits presented in this paper, we use a $\chi^{2}$ fitting procedure, where the value of $\chi_{\min }^{2}$ is distributed as a $\chi^{2}$ distribution with $\mathrm{N}$ degrees of freedom (d.o.f). The fits to the experimental data sets are performed adopting an interval $\chi^{2}-\chi_{\min }^{2}$ corresponding, in the case of normal errors, to the projection of the $\chi^{2}$ hypersurface containing $90 \%$ of probability. This corresponds to $\chi^{2}-\chi_{\min }^{2}=12.02$ 
TABLE II. TOTEM. The values of the Pomeron and secondary Reggeon parameters obtained in global fits to the $\sigma_{\text {tot }}^{p p, \bar{p} p}, \rho^{p p, \bar{p} p}$, and $d \sigma^{p p, \bar{p} p} / d t$ data using Born-level amplitudes. The parameters $\alpha_{+}^{\prime}, \alpha_{-}^{\prime}, r_{+}, r_{-}$, and $a_{1}$ are fixed.

\begin{tabular}{lcccc}
\hline \hline & \multicolumn{4}{c}{ Born-level amplitudes } \\
\cline { 2 - 5 } & BI & BII & BIII & BI + PPP \\
\hline$\epsilon$ & $0.0942 \pm 0.0030$ & $0.0943 \pm 0.0030$ & $0.0949 \pm 0.0032$ & $0.101 \pm 0.012$ \\
$\alpha_{I P}^{\prime}\left(\mathrm{GeV}^{-2}\right)$ & $0.249 \pm 0.014$ & $0.248 \pm 0.014$ & $0.3210 \pm 0.0016$ & $0.175 \pm 0.084$ \\
$\beta_{\mathbb{P}}(0)\left(\mathrm{GeV}^{-1}\right)$ & $1.956 \pm 0.048$ & $1.955 \pm 0.048$ & $1.948 \pm 0.052$ & $2.034 \pm 0.087$ \\
$r_{\mathbb{P}}\left(\mathrm{GeV}^{-2}\right)$ & 5.5 (fixed) & 5.5 (fixed) & $\ldots$ & 5.5 (fixed) \\
$\eta_{+}$ & $0.338 \pm 0.050$ & $0.338 \pm 0.050$ & $0.333 \pm 0.053$ & $0.310 \pm 0.061$ \\
$\beta_{+}(0)\left(\mathrm{GeV}^{-1}\right)$ & $3.73 \pm 0.40$ & $3.73 \pm 0.40$ & $3.71 \pm 0.42$ & $3.80 \pm 0.40$ \\
$\eta_{-}$ & $0.529 \pm 0.085$ & $0.529 \pm 0.085$ & $0.528 \pm 0.089$ & $0.524 \pm 0.089$ \\
$\beta_{-}(0)\left(\mathrm{GeV}^{-1}\right)$ & $2.90 \pm 0.52$ & $2.90 \pm 0.52$ & $2.90 \pm 0.54$ & $2.88 \pm 0.53$ \\
$a_{1}\left(\mathrm{GeV}^{2}\right)$ & $\cdots$ & $m_{\rho}^{2}$ (fixed) & $m_{\rho}^{2}$ (fixed) & $\ldots$ \\
$a_{2}\left(\mathrm{GeV}^{2}\right)$ & $\ldots$ & $\cdots$ & $1.79 \pm 0.44$ & $\ldots$ \\
$\beta_{\mathbb{P P}}(0)\left(\mathrm{GeV}^{-1}\right)$ & $\ldots$ & $\cdots$ & $\ldots$ & $1.52 \pm 0.66$ \\
$\chi^{2} / \mathrm{d}$.o.f & 0.65 & 0.65 & 0.65 & 0.63 \\
\hline \hline
\end{tabular}

and 13.36 in the case of seven and eight free parameters, respectively. Following the philosophy of using the minimum number of free parameters, in the following analyses, the slopes of the secondary-Reggeon linear trajectories, $\alpha_{+}^{\prime}$ and $\alpha_{-}^{\prime}$, are fixed at $0.9 \mathrm{GeV}^{-1}$. These values are in agreement with those usually obtained in ChewFrautschi plots. Also, the slopes associated with the form factors of the secondary Reggeons are fixed at $r_{+}=r_{-}=4.0 \mathrm{GeV}^{-2}$. These parameters have very little statistical correlation with the Pomeron parameters, and
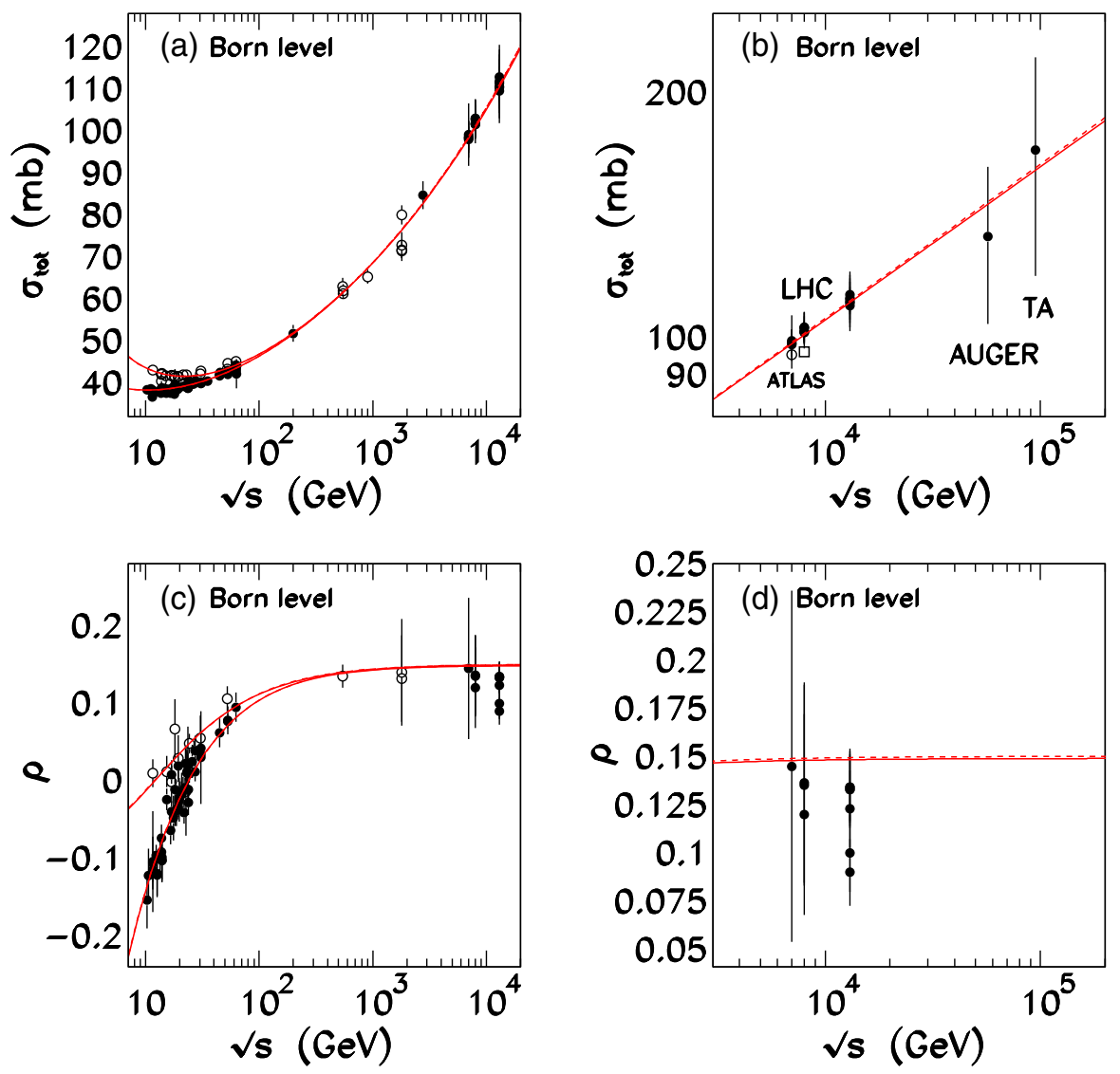

FIG. 2. Total cross section [(a) and (b)] and ratio of the real to imaginary part of the forward scattering amplitude [(c) and (d)] for $p p$ $(\bullet)$ and $\bar{p} p(\circ)$ channels. The solid line shows the results obtained using the BI or BII or BI $+\mathbb{P P P}$ models, while the dashed line shows the results obtained using the BIII model. Also shown the predictions for cosmic ray energies. 

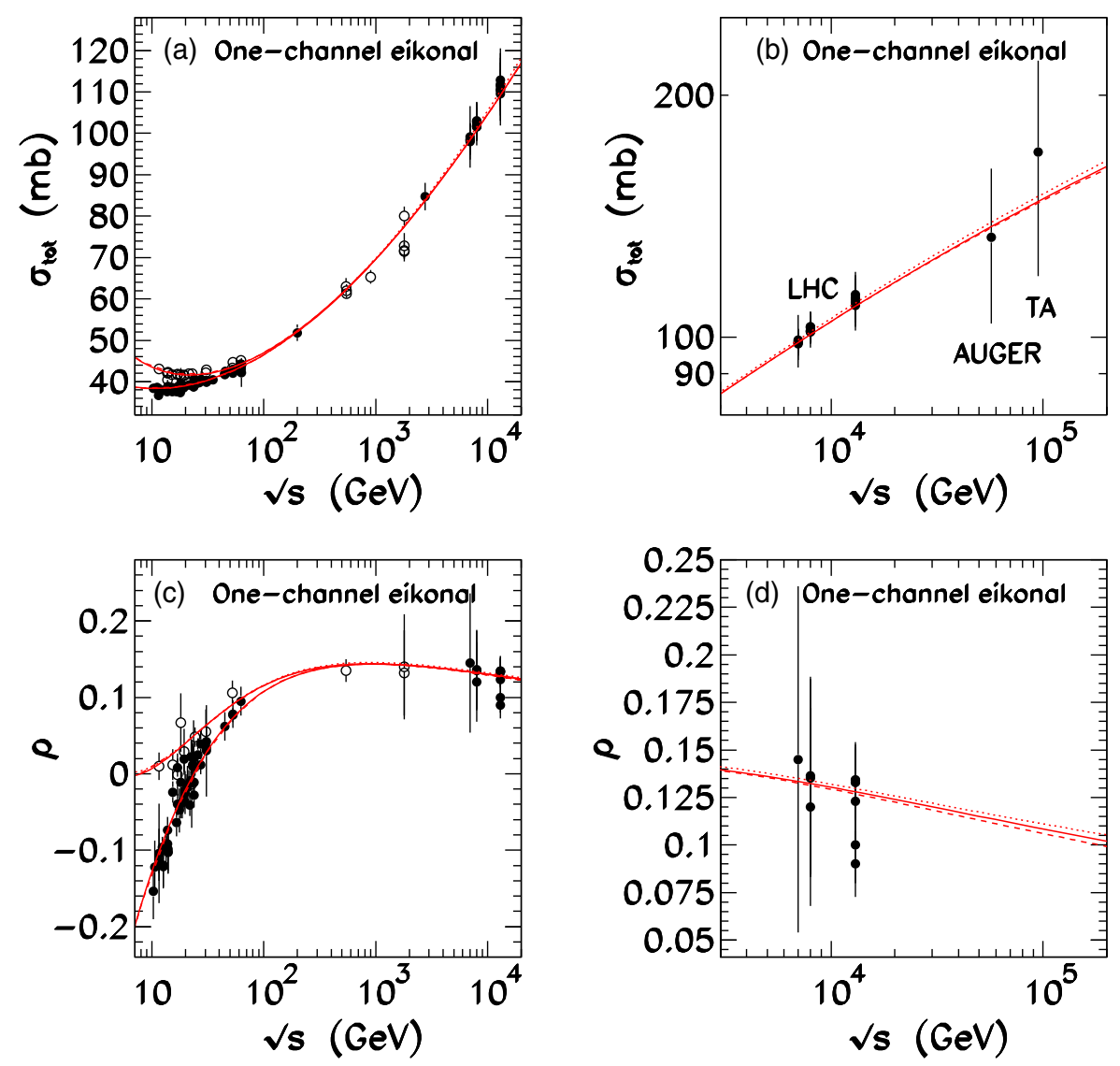

FIG. 3. Total cross section [(a) and (b)] and ratio of the real to imaginary part of the forward scattering amplitude [(c) and (d)] for $p p$ $(\bullet)$ and $\bar{p} p(\circ)$ channels. The solid, dashed, and dotted lines show the results obtained using the OI, OII, and OIII models, respectively. Also shown the predictions for cosmic ray energies.

their fixed values are consistent with those obtained in previous studies $[15,21,22]$. We also fix the scale of the pion-Pomeron vertex at $a_{1}=m_{\rho}^{2}=(0.776 \mathrm{GeV})^{2}$ [23].

In the case of Born-level amplitudes, the values of the Regge parameters determined by global fits to $p p$ and $\bar{p} p$ data are listed in Table II. Notice that in the case of $\mathrm{BI}, \mathrm{BII}$, and $\mathrm{BI}+\mathbb{P P}$ models we fixed the parameter $r_{\mathbb{P}}$ at $5.5 \mathrm{GeV}^{-2}$, which corresponds to the slope of the electromagnetic proton form factor. As discussed in Ref. [20], it is the natural choice for the computation of double-diffractive central Higgs production via $W W$ fusion (since the $W$ boson is radiated from a quark, like the photon). Moreover, our analyses show that at this $r_{\mathbb{P}}$ value the Pomeron is described by trajectories with $\alpha_{\mathbb{P}}^{\prime} \simeq 0.25 \mathrm{GeV}^{-2}$ (see Table II). Interestingly enough, these values for $\alpha_{\mathbb{P}}^{\prime}$ are consistent with the ones recently obtained from holographic QCD models [47]. Furthermore, if we perform the global fits at another value of $r_{\mathbb{P}}$, say $4.0 \mathrm{GeV}^{-2}$ (which is not atypical [20]), we obtain the values $\alpha_{\mathbb{P}}^{\prime}=0.3346 \pm 0.0085 \mathrm{GeV}^{-2}$, $0.3339 \pm 0.0085 \mathrm{GeV}^{-2}$, and $\alpha_{\mathbb{P}}^{\prime}=0.3346 \pm 0.0090 \mathrm{GeV}^{-2}$ in the case of BI, BII, and BI $+\mathbb{P} P$ models, respectively, while the remaining free parameters follow with the same values. The parameters obtained in the analyses with
$\mathrm{BI}, \mathrm{BII}$, and $\mathrm{BI}+\mathbb{P P}$ models are very close to each other and the description of the data resulted in substantially the same curves, shown in Fig. 2 (solid curves). The dashed curves in the same figure are for the BIII model. Figures 2-4 have the same layout: the part (a) shows the $\sigma_{\text {tot }}^{p p, \bar{p} p}$ accelerator data, the part (b) extends the range in $\sqrt{s}$ of the part (a), the part (c) shows the $\rho^{p p, \bar{p} p}$ data, and the part (d) extends the range in $\sqrt{s}$ of the part (c). For comparison purposes, we have included in part (b) of these figures estimates of $\sigma_{\text {tot }}^{p p}$ obtained from cosmic ray experiments, namely, the AUGER experimental result at $\sqrt{s}=57 \mathrm{TeV}[48]$ and the Telescope Array result at $\sqrt{s}=$ $95 \mathrm{TeV}$ [49].

The preceding results using Born-level amplitudes have demonstrated that it is possible a good description of forward data up to LHC energy by using a constant value of $\epsilon$; even so, from the Table II, we see that the ratio of two-Pomeron to one-Pomeron exchange couplings is not so small,

$$
R \equiv \frac{\left|\mathbb{P} \mathbb{P}_{\text {coupling }}\right|}{\mathbb{P}_{\text {coupling }}}=\frac{\beta_{\mathbb{P} P}^{2}(0)}{\beta_{\mathbb{P}}^{2}(0)} \simeq 0.6,
$$



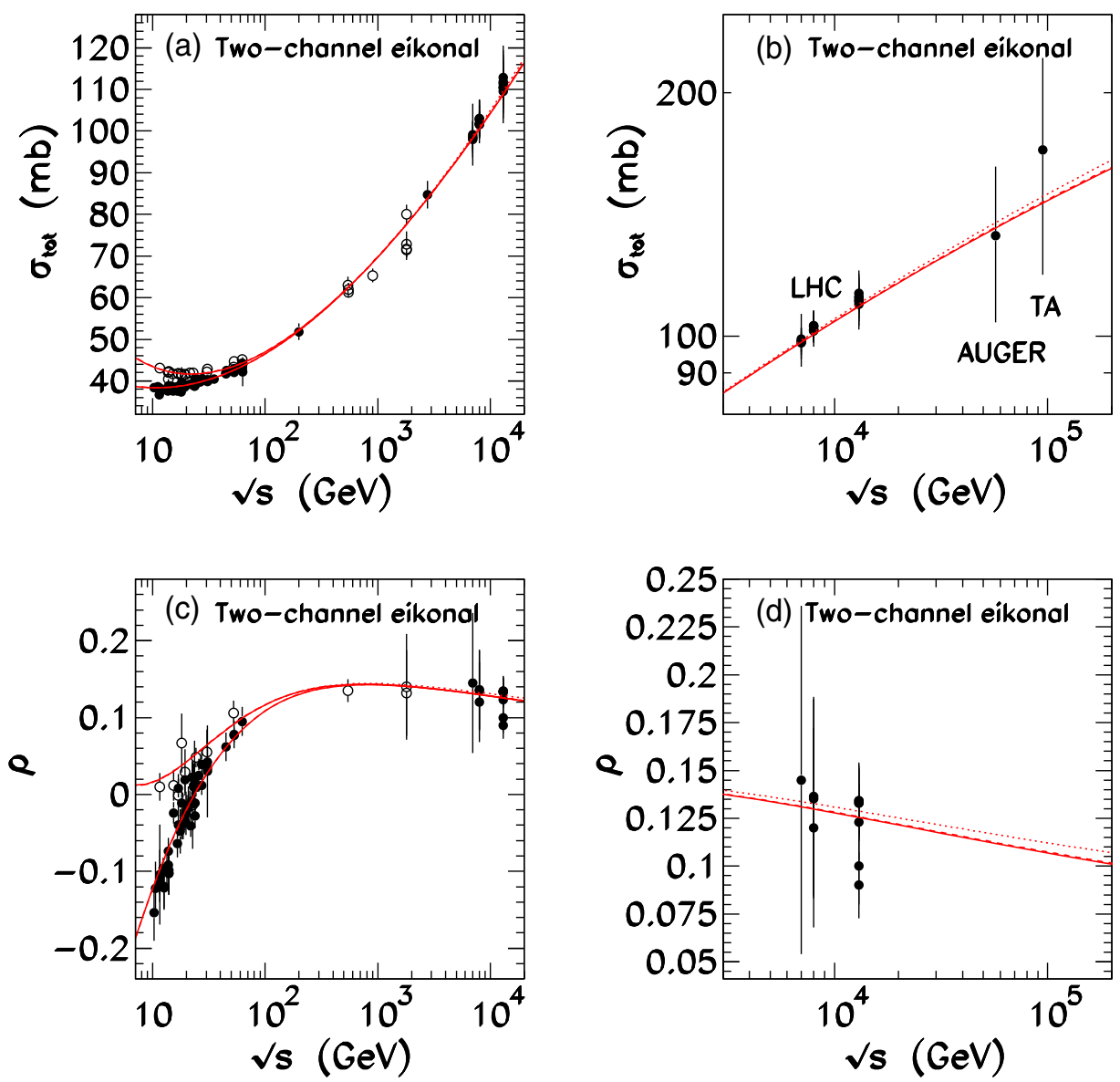

FIG. 4. Total cross section [(a) and (b)] and ratio of the real to imaginary part of the forward scattering amplitude [(c) and (d)] for $p p$ $(\bullet)$ and $\bar{p} p(\circ)$ channels. The solid, dashed, and dotted lines show the results obtained using the TI, TII, and TIII models, respectively. Also shown the predictions for cosmic ray energies.

which suggests the violation of unitarity at the presently available energies. Therefore, contrary to the expectation that the violation of unitarity would occur only far above the LHC energies, the value of $R$ indicates that the unitarization breaking is a current problem. Most importantly, this result indicates that multi-Pomeron exchanges

TABLE III. TOTEM. The values of the Pomeron and secondary Reggeon parameters obtained in global fits to the $\sigma_{\text {tot }}^{p p, \bar{p} p}, \rho^{p p, \bar{p} p}$, and $d \sigma^{p p, \bar{p} p} / d t$ data using one-channel eikonalized amplitudes. The parameters $\alpha_{+}^{\prime}, \alpha_{-}^{\prime}, r_{+}, r_{-}$, and $a_{1}$ are fixed.

\begin{tabular}{lccc}
\hline \hline & \multicolumn{3}{c}{ Eikonalized amplitudes (one-channel eikonal) } \\
\cline { 2 - 4 } & OI & OII & OIII \\
\hline$\epsilon$ & $0.1258 \pm 0.0014$ & $0.1267 \pm 0.0049$ & $0.1309 \pm 0.0087$ \\
$\alpha_{I P}^{\prime}\left(\mathrm{GeV}^{-2}\right)$ & $0.066 \pm 0.012$ & $0.039 \pm 0.012$ & $0.052 \pm 0.029$ \\
$\beta_{\mathbb{P}}(0)\left(\mathrm{GeV}^{-1}\right)$ & $1.811 \pm 0.010$ & $1.795 \pm 0.035$ & $1.77 \pm 0.12$ \\
$r_{\mathbb{P}}\left(\mathrm{GeV}^{-2}\right)$ & $6.53 \pm 0.23$ & $7.0 \pm 5.2$ & $\ldots$ \\
$\eta_{+}$ & $0.278 \pm 0.063$ & $0.275 \pm 0.060$ & $0.267 \pm 0.056$ \\
$\beta_{+}(0)\left(\mathrm{GeV}^{-1}\right)$ & $3.98 \pm 0.47$ & $3.95 \pm 0.70$ & $3.94 \pm 0.40$ \\
$\eta_{-}$ & $0.534 \pm 0.087$ & $0.531 \pm 0.088$ & $0.531 \pm 0.087$ \\
$\beta_{-}(0)\left(\mathrm{GeV}^{-1}\right)$ & $3.41 \pm 0.64$ & $3.39 \pm 0.71$ & $3.40 \pm 0.62$ \\
$a_{1}\left(\mathrm{GeV}^{2}\right)$ & $\cdots$ & $m_{\rho}^{2}$ (fixed) & $\ldots .$. \\
$a_{2}\left(\mathrm{GeV}^{2}\right)$ & $\ldots$ & $\cdots$ & $0.58 \pm 0.26$ \\
$\chi^{2} /$ d.o.f & 0.66 & 0.66 & 0.66 \\
\hline \hline
\end{tabular}


TABLE IV. TOTEM. The values of the Pomeron and secondary Reggeon parameters obtained in global fits to the $\sigma_{\mathrm{tot}}^{p p, \bar{p} p}, \rho^{p p, \bar{p} p}$, and $d \sigma^{p p, \bar{p} p} / d t$ data using two-channel eikonalized amplitudes. The parameters $\alpha_{+}^{\prime}, \alpha_{-}^{\prime}, r_{+}, r_{-}$, and $a_{1}$ are fixed.

\begin{tabular}{lccc}
\hline \hline & \multicolumn{3}{c}{ Eikonalized amplitudes (two-channel eikonal) } \\
\cline { 2 - 4 } & TI & TII & TIII \\
\hline$\epsilon$ & $0.152 \pm 0.010$ & $0.1513 \pm 0.0017$ & $0.1544 \pm 0.0073$ \\
$\alpha_{I P}^{\prime}\left(\mathrm{GeV}^{-2}\right)$ & $0.0460 \pm 0.0085$ & $0.048 \pm 0.023$ & $0.0500 \pm 0.0053$ \\
$\beta_{\mathbb{P}}(0)\left(\mathrm{GeV}^{-1}\right)$ & $1.72 \pm 0.16$ & $1.726 \pm 0.023$ & $1.75 \pm 0.12$ \\
$r_{\mathbb{P}}\left(\mathrm{GeV}^{-2}\right)$ & $5.7 \pm 1.2$ & $5.67 \pm 0.39$ & $\ldots$ \\
$\eta_{+}$ & $0.257 \pm 0.058$ & $0.258 \pm 0.014$ & $0.261 \pm 0.053$ \\
$\beta_{+}(0)\left(\mathrm{GeV}^{-1}\right)$ & $4.27 \pm 0.44$ & $4.28 \pm 0.13$ & $4.32 \pm 0.42$ \\
$\eta_{-}$ & $0.537 \pm 0.086$ & $0.535 \pm 0.073$ & $0.538 \pm 0.086$ \\
$\beta_{-}(0)\left(\mathrm{GeV}^{-1}\right)$ & $3.80 \pm 0.69$ & $3.79 \pm 0.59$ & $3.83 \pm 0.69$ \\
$a_{1}\left(\mathrm{GeV}^{2}\right)$ & $\cdots$ & $m_{\rho}^{2}$ (fixed) & $\ldots$ \\
$a_{2}\left(\mathrm{GeV}^{2}\right)$ & $\ldots$ & $\cdots$ & $1.02 \pm 0.13$ \\
$\chi^{2} /$ d.o.f & 0.71 & 0.71 & 0.70 \\
\hline \hline
\end{tabular}

must be included in order to restore unitarity. Multiple exchanges can somehow be taken into account in an appropriate unitarization scheme, such as the eikonalization one, as already discussed.

The values of the Regge parameters obtained using onechannel eikonal models are listed in Table III. We see that all eikonalized fits to the data prefer very small values of $\alpha_{I P}^{\prime}$, in the case of OI, OII, and OIII models these values are, respectively, $\alpha_{I P}^{\prime}=0.066 \pm 0.012, \quad 0.039 \pm 0.012$, and $0.052 \pm 0.029 \mathrm{GeV}^{-2}$. Thus, after the eikonalization, the Pomeron looks similar to a fixed pole at $\alpha_{I P}(0)=1$. We can see that the effect of the pion-loop insertions further decreases the values of $\alpha_{I P}^{\prime}$ : the result for OII (OIII) model is about a factor 1.7 (1.3) lower than the OI one. We also note a substantial increase of the Pomeron intercept in relation to Born-level models: the $\epsilon$ parameters are increased by about $34 \%$ for OI and OII models and by about $38 \%$ for the OIII model. The curves of $\sigma_{\text {tot }}(s)$ and $\rho(s)$ for the one-channel models, compared with the experimental data, are shown in Fig. 3.

The same significant increase of the Pomeron intercept in relation to Born-level models is observed in the twochannel eikonal models, as shown in Table IV. In the case of TI, TII, and TIII models, the increase is even greater, with the $\epsilon$ parameters being about $61 \%, 60 \%$, and $53 \%$ higher, respectively, when compared to the same parameters in models with Born-level amplitudes. Again, we see that the eikonal fits to the data prefer very small values of $\alpha_{I P}^{\prime}$, but now with values of $\alpha_{I P}^{\prime}$ closer to each other. In the case of TI, TII, and TIII models, these values are, respectively, $\alpha_{I P}^{\prime}=0.046 \pm 0.012, \quad 0.048 \pm 0.012$, and $0.050 \pm 0.029 \mathrm{GeV}^{-2}$. The results for $\sigma_{\text {tot }}(s)$ and $\rho(s)$, using two-channel eikonal amplitudes, are shown in Fig. 4.

For both one- and two-channel eikonal models, the values of the parameter $r_{\mathbb{P}}$ are insensitive to changes in the form of the Pomeron trajectory, as they are of the same order. In the case of one-channel models, the change from a linear to a nonlinear Pomeron trajectory leads to an increase of $r_{\mathbb{P}}$ of only $7 \%$, while in two-channel models the same change leaves the value of $r_{\mathbb{P}}$ practically unchanged. In Table $\mathrm{V}$, we show the predictions on the high energy total cross section and $\rho$ parameter, made using the BIII, OIII, and TIII models. A comparison among the cross sections and $\rho$ parameters at high energies, from the models BIII (solid), OIII (dashed), and TIII (dotted), is shown in Fig. 5.

TABLE V. Predictions and uncertainties for the forward scattering quantities $\sigma_{\text {tot }}^{p p}$ and $\rho^{p p}$ using different Regge models. The uncertainties are just estimates since the full covariance matrix was not employed.

\begin{tabular}{|c|c|c|c|c|c|c|}
\hline \multirow[b]{2}{*}{$\sqrt{s}(\mathrm{TeV})$} & \multicolumn{2}{|c|}{ BIII model } & \multicolumn{2}{|c|}{ OIII model } & \multicolumn{2}{|c|}{ TIII model } \\
\hline & $\sigma_{\text {tot }}(\mathrm{mb})$ & $\rho$ & $\sigma_{\text {tot }}(\mathrm{mb})$ & $\rho$ & $\sigma_{\text {tot }}(\mathrm{mb})$ & $\rho$ \\
\hline 7.0 & $98.7 \pm 2.7$ & $0.149 \pm 0.008$ & $99.2 \pm 2.7$ & $0.135 \pm 0.008$ & $98.8 \pm 2.7$ & $0.134 \pm 0.008$ \\
\hline 8.0 & $101.2 \pm 2.8$ & $0.149 \pm 0.009$ & $101.5 \pm 2.8$ & $0.134 \pm 0.009$ & $101.1 \pm 2.8$ & $0.133 \pm 0.010$ \\
\hline 13.0 & $110.9 \pm 3.3$ & $0.150 \pm 0.009$ & $110.2 \pm 3.3$ & $0.130 \pm 0.009$ & $109.6 \pm 3.3$ & $0.129 \pm 0.012$ \\
\hline 57.0 & $148 \pm 8$ & $0.150 \pm 0.018$ & $139 \pm 7$ & $0.116 \pm 0.018$ & $138.1 \pm 7$ & $0.117 \pm 0.018$ \\
\hline 95.0 & $162 \pm 12$ & $0.150 \pm 0.026$ & $150 \pm 11$ & $0.112 \pm 0.026$ & $149 \pm 11$ & $0.113 \pm 0.026$ \\
\hline
\end{tabular}



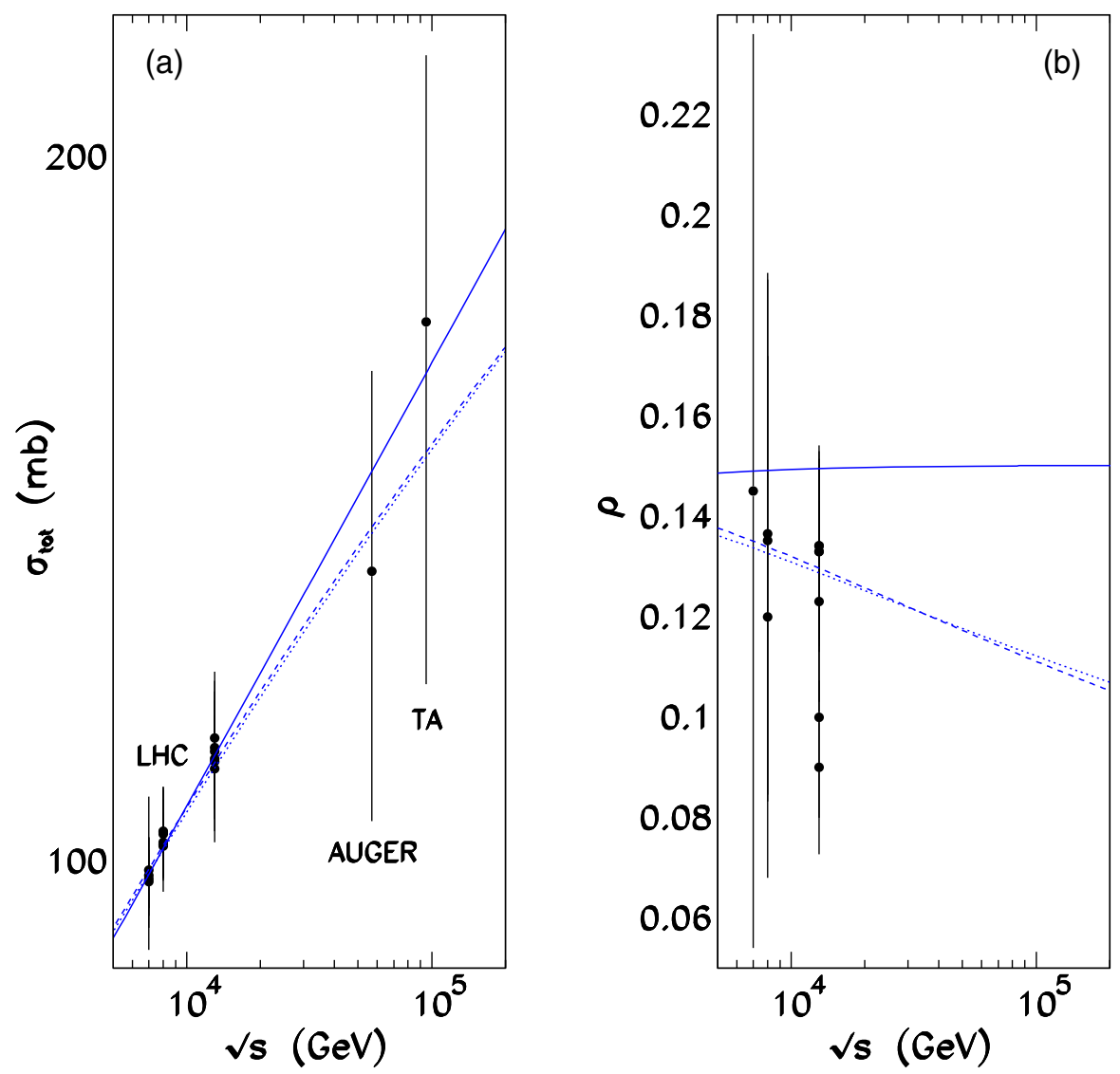

FIG. 5. Total cross section [part (a)] and ratio of the real to imaginary part of the forward scattering amplitude [part (b)] for $p p(\bullet)$ channel. The solid, dashed, and dotted lines show the results obtained using the BIII, OIII, and TIII models, respectively. Also shown the predictions for cosmic ray energies.

In Fig. 6, we show the description of the elastic $p p$ differential cross section data for all models discussed so far. We see that in all cases the data in the range $|t|_{\min } \leq$ $|t| \leq 0.1 \mathrm{GeV}^{2}$ are well described, where we have adopted $|t|_{\min } \sim 10|t|_{\text {int }}$, since in the region $|t| \gg|t|_{\text {int }}$ the nuclear scattering dominates. The value of $|t|_{\text {int }}$ (where the interference between the Coulomb and hadronic amplitudes is of maximum significance) can be simply determined from the practical relation $|t|_{\text {int }}=0.071 / \sigma_{\text {tot }}$ [50]. In Figs. 6(a)-6(c), we show the curves of $d \sigma^{p p} / d t$ for the Born level, one- and two-channel eikonal models, respectively. We can see that it is not possible to distinguish between different models of the Pomeron since the curves representing models of types I (solid), II (dashed), and III (dotted) fall on very nearly the same curve as the model of type I. A comparison among the differential cross sections from the models BIII (solid), OIII (dashed), and TIII (dotted) is shown in Fig. 6(d).

In order to see more clearly the effects of the correlation information on the data sets, we compare, in Fig. 2(b), the total $p p$ cross section data of TOTEM with the ATLAS data. The ATLAS results include the luminosity-dependent measurements at $\sqrt{s}=7 \mathrm{TeV}, \sigma_{\mathrm{tot}}^{p p}=95.35 \pm 1.36$ [51] [open circle in Fig. 2(b)], and $\sqrt{s}=8 \mathrm{TeV}, \sigma_{\mathrm{tot}}^{p p}=96.07 \pm$ 0.92 [52] [open square in Fig. 2(b)]. These measurements rely on the optical theorem. The luminosity, necessary to normalize the elastic cross section, is determined from LHC beam parameters using van der Meer scans [53]. Before the introduction of correlation information, we recognize some tension between the TOTEM and ATLAS measurements. For example, if we compare the ATLAS result for $\sigma_{\text {tot }}^{p p}$ at $\sqrt{s}=7 \mathrm{TeV}$ with, say, the value $\sigma_{\mathrm{tot}}^{p p}=98.6 \pm 2.2$ measured by TOTEM at the same energy, the difference between the values corresponds to $1.5 \sigma$. In the same way, if we compare the ATLAS result for $\sigma_{\mathrm{tot}}^{p p}$ at $\sqrt{s}=8 \mathrm{TeV}$ with the highest value obtained by TOTEM in the same energy, $\sigma_{\mathrm{tot}}^{p p}=103.0 \pm 2.3$, the difference goes up to $3 \sigma$. However, after the inclusion of the correlated systematics that allows treating each result as independent, the difference between the ATLAS results and the average values of the TOTEM measurements and uncertainties at 7 and $8 \mathrm{TeV}$ drops to 0.7 and $1.6 \sigma$, respectively. We can therefore ameliorate the tension between the TOTEM and ATLAS measurements by introducing correlation information.

In summary, the paper is devoted to a detailed study of the soft Pomeron. More precisely, we evaluate the relative 

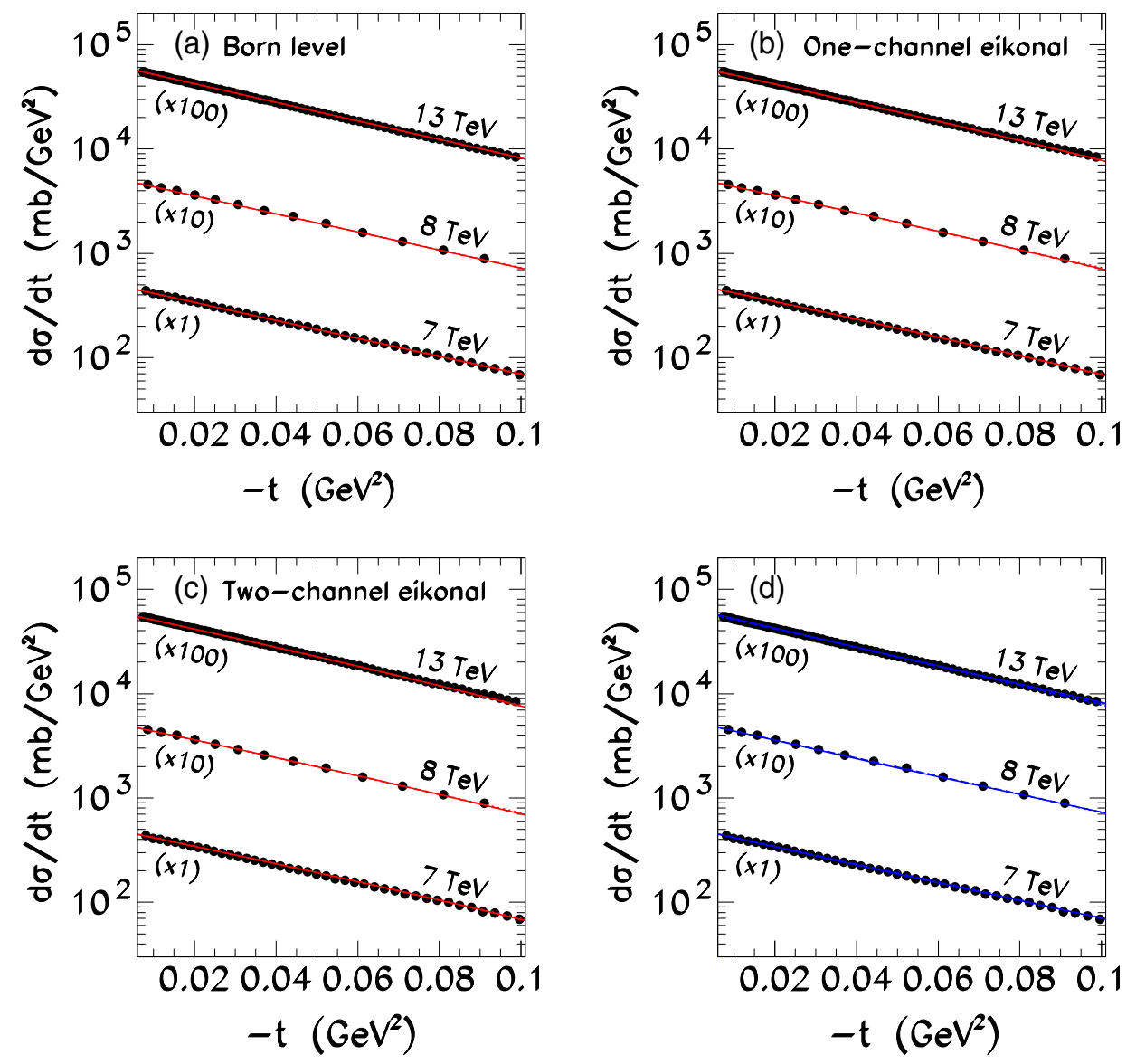

FIG. 6. The elastic differential cross section for $p p(\bullet)$ channel. In parts (a)-(c), we show the curves of $d \sigma^{p p} / d t$ for the Born level, oneand two-channel eikonal models, respectively. A comparison among the differential cross sections from the models BIII (solid), OIII (dashed), and TIII (dotted) is shown in part (d).

plausibility of different combinations of vertices and trajectories of the soft Pomeron in the light of the recent LHC data, taking into account the existent systematic correlation among them. The methods used in this paper for the examination of the Pomeron properties can also be applied to obtain constraints on the Odderon parameters. Work in this direction is in progress.

\section{ACKNOWLEDGMENTS}

This research was partially supported by the Agencia Nacional de Investigación e Innovación under the Project No. ANII-FCE-126412 and by the Conselho Nacional de Desenvolvimento Científico e Tecnológico under Grants No. 141496/2015-0 and No. 155628/2018-6.
[1] G. Antchev et al., Eur. Phys. J. C 79, 785 (2019).

[2] A. A. Godizov, Phys. Rev. D 101, 074028 (2020); E. Ferreira, A. K. Kohara, and T. Kodama, arXiv:2011 .13335; L. A. Harland-Lang, V. A. Khoze, A. D. Martin, and M. G. Ryskin, Phys. Rev. D 99, 034011 (2019); M. Broilo, D. A. Fagundes, E. G. S. Luna, and M. J. Menon, Phys. Lett. B 799, 135047 (2019); Eur. Phys. J. C 79, 1033 (2019); T. Csörgő, R. Pasechnik, and A. Ster, Eur. Phys. J. C 79, 62 (2019); T. Csörgő, T. Novak, R. Pasechnik, A. Ster, and I. Szanyi, arXiv:1912.11968; V. P. Gonçalves and P. V.
R. G. Silva, Eur. Phys. J. C 79, 237 (2019); E. Martynov and B. Nicolescu, Phys. Lett. B 778, 414 (2018); E. Martynov and B. Nicolescu, Phys. Lett. B 786, 207 (2018); M. Broilo, E. G. S. Luna, and M. J. Menon, Phys. Lett. B 781, 616 (2018); V. A. Khoze, A. D. Martin, and M. G. Ryskin, Phys. Lett. B 780, 352 (2018); S. M. Troshin and N. E. Tyurin, Mod. Phys. Lett. A 33, 1850206 (2018); V. A. Khoze, A. D. Martin, and M. G. Ryskin, Phys. Lett. B 784, 192 (2018); 787, 167 (2018); Phys. Rev. D 97, 034019 (2018); P. Lebiedowicz, O. Nachtmann, and A. Szczurek, Phys. 
Rev. D 98, 014001 (2018); E. Gotsman, E. Levin, and I. Potashnikova, Phys. Lett. B 786, 472 (2018); M. Broilo, E. G. S. Luna, and M. J. Menon, Phys. Rev. D 98, 074006 (2018); W. Broniowski, L. Jenkovszky, E. Ruiz Arriola, and I. Szanyi, Phys. Rev. D 98, 074012 (2018); Y. M. Shabelski and A. G. Shuvaev, Eur. Phys. J. C 78, 497 (2018); L. Jenkovszky, I. Szanyi, and C. I. Tan, Eur. Phys. J. A 54, 116 (2018); E. Ferreira, A. K. Kohara, and J. Sesma, Phys. Rev. D 98, 094029 (2018).

[3] D. Joynson, E. Leader, B. Nicolescu, and C. Lopez, Nuovo Cimento A 30, 345 (1975); L. Lukaszuk and B. Nicolescu, Lett. Nuovo Cimento 8, 405 (1973).

[4] Y. V. Kovchegov and E. Levin, Quantum Choromodynamics at High Energies (Cambridge University Press, Cambridge, United Kingdom, 2012).

[5] C. Contreras, E. Levin, R. Meneses, and M. Sanhueza, Phys. Rev. D 101, 096019 (2020); J. Bartels, C.Contreras, and G. P. Vacca, J. High Energy Phys. 04 (2020) 183; Y. V. Kovchegov, L. Szymanowski, and S. Wallon, Phys. Lett. B 586, 267 (2004); J. Bartels, L. N. Lipatov, and G. P. Vacca, Phys. Lett. B 477, 178 (2000); J. Bartels, C. Contreras, and G. P. Vacca, Phys. Rev. D 95, 014013 (2017); J. High Energy Phys. 03 (2016) 201.

[6] V. V. Ezhela, V. A. Petrov, and N. P. Tkachenko, arXiv: 2003.03817.

[7] S. Pacetti, Y. Srivastava, and G. Pancheri, Phys. Rev. D 99, 034014 (2019).

[8] G. Pancheri and Y. N. Srivastava, Eur. Phys. J. C 77, 150 (2017); D. A. Fagundes, A. Grau, S. Pacetti, G. Pancheri, and Y. N. Srivastava, Phys. Rev. D 88, 094019 (2013).

[9] P. A. Zyla et al. (Particle Data Group), Prog. Theor. Exp. Phys. 2020, 083C01 (2020), https://pdg.lbl.gov/2020/html/ authors_2020.html.

[10] V. N. Gribov, Zh. Eksp. Teor. Fiz. 42, 1260 (1962) [Sov. Phys. JETP 15, 873 (1962)]; Zh. Eksp. Teor. Fiz. 53, 654 (1967) [Sov. Phys. JETP 26, 414 (1968)]; Yadernaya fizika 9, 640 (1969) [Sov. J. Nucl. Phys. 9, 369 (1969)].

[11] M. Baker and K. A. Ter-Martirosyan, Phys. Rep. 28, 1 (1976).

[12] V. Barone and E. Predazzi, High-Energy Particle Diffraction (Springer-Verlag, Berlin, 2002); S. Donnachie, G. Dosch, P. Landshoff, and O. Nachtmann, Pomeron Physics and QCD (Cambridge University Press, Cambridge, United Kingdom, 2002); J. R. Forshaw and D. A. Ross, Quantum Chromodynamics and the Pomeron (Cambridge University Press, Cambridge, United Kingdom, 1997); P. D. B. Collins, An Introduction to Regge Theory and High Energy Physics (Cambridge University Press, Cambridge, United Kingdom, 1977).

[13] A. Donnachie and P. V. Landshoff, Phys. Lett. B 296, 227 (1992).

[14] J. R. Cudell, K. Kang, and S. K. Kim, Phys. Lett. B 395, 311 (1997).

[15] R. J. M. Covolan, J. Montanha, and K. Goulianos, Phys. Lett. B 389, 176 (1996).

[16] E. G. S. Luna, M. J. Menon, and J. Montanha, Nucl. Phys. A745, 104 (2004); Braz. J. Phys. 34, 268 (2004); E. G. S. Luna and M. J. Menon, Phys. Lett. B 565, 123 (2003).

[17] R. F. Ávila, E. G. S. Luna, and M. J. Menon, Phys. Rev. D 67, 054020 (2003); J. R. Cudell, V. V. Ezhela, P. Gauron,
K. Kang, Yu. V. Kuyanov, S. B. Lugovsky, E. Martynov, B. Nicolescu, E. A. Razuvaev, and N. P. Tkachenko, Phys. Rev. Lett. 89, 201801 (2002).

[18] J. Breitweg et al., Eur. Phys. J. C 1, 81 (1998); T. Ahmed et al., Phys. Lett. B 299, 374 (1993); M. Derrick et al., Phys. Lett. B 293, 465 (1992).

[19] A. A. Anselm and V. N. Gribov, Phys. Lett. B 40, 487 (1972).

[20] V. A. Khoze, A. D. Martin, and M. G. Ryskin, Eur. Phys. J. C 18, 167 (2000).

[21] E. G. S. Luna, V. A. Khoze, A. D. Martin, and M. G. Ryskin, Eur. Phys. J. C 59, 1 (2009).

[22] E. G. S. Luna, V. A. Khoze, A. D. Martin, and M. G. Ryskin, Eur. Phys. J. C 69, 95 (2010).

[23] V. A. Khoze, A. D. Martin, and M. G. Ryskin, Nucl. Phys. B, Proc. Suppl. 99, 213 (2001).

[24] V. A. Petrov and V. A. Okorokov, Int. J. Mod. Phys. A 33, 1850077 (2018); V. A. Okorokov and S. D. Campos, Int. J. Mod. Phys. A 32, 1750175 (2017); S. D. Campos and V. A. Okorokov, Int. J. Mod. Phys. A 25, 5333 (2010).

[25] A. Donnachie and P. V. Landshoff, Phys. Lett. B 727, 500 (2013).

[26] A. Martin, Phys. Rev. 129, 1432 (1963); M. Froissart, Phys. Rev. 123, 1053 (1961).

[27] M. Giffon, E. Martynov, and E. Predazzi, Z. Phys. C 76, 155 (1997).

[28] S. M. Troshin and N. E. Tyurin, Phys. Part. Nucl. 30, 550 (1999); Eur. Phys. J. A 53, 57 (2017); A. N. Vall, L. L. Jenkovszky, and B. V. Struminsky, Fiz. Elem. Chastits At. Yadra 19, 180 (1988) [Sov. J. Part. Nucl. 19, 77 (1988)]; V. I. Savrin, N. E. Tyurin, and O. A. Khrustalev, Fiz. Elem. Chastits At. Yadra 7, 21 (1976) [Part. Nuclei 7, 21 (1976)].

[29] M. L. Good and W. D. Walker, Phys. Rev. 120, 1857 (1960).

[30] P. Lipari and M. Lusignoli, Eur. Phys. J. C 73, 2630 (2013); Phys. Rev. D 80, 074014 (2009).

[31] M. Broilo, V. P. Gonçalves, and P. V. R. G. Silva, Phys. Rev. D 101, 074034 (2020); Int. J. Mod. Phys. A 35, 2050127 (2020).

[32] V. A. Khoze, A. D. Martin, and M. G. Ryskin, Eur. Phys. J. C 14, 525 (2000).

[33] E. Gotsman, E. Levin, and U. Maor, Phys. Lett. B 452, 387 (1999).

[34] E. Gotsman, E. Levin, and U. Maor, Phys. Lett. B 309, 199 (1993).

[35] G. Antchev et al., Europhys. Lett. 96, 21002 (2011).

[36] G. Antchev et al., Europhys. Lett. 101, 21002 (2013).

[37] G. Antchev et al., Europhys. Lett. 101, 21004 (2013).

[38] G. Antchev et al., Phys. Rev. Lett. 111, 012001 (2013).

[39] G. Antchev et al., Eur. Phys. J. C 76, 661 (2016).

[40] G. Antchev et al., Europhys. Lett. 95, 41001 (2011).

[41] G. Antchev et al., Nucl. Phys. B899, 527 (2015).

[42] G. Antchev et al., Eur. Phys. J. C 79, 861 (2019).

[43] CMS Collaboration, Report No. CMS-PAS-EWK-10-004, 2010; CMS Collaboration, Report No. CMS-DP-2011000C, 2011.

[44] G. Antchev et al., Eur. Phys. J. C 79, 103 (2019).

[45] A. Martin, Phys. Lett. B 404, 137 (1997).

[46] R. J. N. Philips and V. D. Barger, Phys. Lett. 46B, 412 (1973). 
[47] D. M. Rodrigues, E. F. Capossoli, and H. Boschi-Filho, Phys. Rev. D 95, 076011 (2017); A. Ballon-Bayona, R. C. Quevedo, M. S. Costa, and M. Djurić, Phys. Rev. D 93, 035005 (2016); E. F. Capossoli, D. Li, and H. Boschi-Filho, Eur. Phys. J. C 76, 320 (2016); Phys. Lett. B 760, 101 (2016); E. F. Capossoli and H. Boschi-Filho, Phys. Lett. B 753, 419 (2016); Phys. Rev. D 88, 026010 (2013).

[48] P. Abreu et al., Phys. Rev. Lett. 109, 062002 (2012).
[49] R. U. Abbasi et al., Phys. Rev. D 92, 032007 (2015).

[50] M. M. Block and R. N. Cahn, Rev. Mod. Phys. 57, 563 (1985).

[51] G. Aad et al., Nucl. Phys. B889, 486 (2014).

[52] M. Aaboud et al., Phys. Lett. B 761, 158 (2016).

[53] S. van der Meer, Report No. ISR-PO-68-31, 1968, http://cds .cern.ch/record/296752. 